\title{
Análise da vulnerabilidade em saúde no estado do Ceará
}

A saúde exerce um papel de extrema importância para a promoção do desenvolvimento sustentável e redução da vulnerabilidade social, sendo em grande parte determinada por fatores ambientais, econômicos e sociais. Este artigo tem como objetivo geral analisar a vulnerabilidade dos municípios cearenses em relação à saúde através da elaboração de um Índice de Vulnerabilidade em Saúde (IVS). Os objetivos específicos são: caracterizar o estado do Ceará com relação a indicadores de vulnerabilidade em saúde, formados a partir das variáveis selecionadas para o estudo e calcular o IVS, de modo a possibilitar o agrupamento e a hierarquização dos municípios com relação a este tipo de vulnerabilidade. Os dados foram obtidos através do DATASUS, Instituto de Pesquisa e Estratégia Econômica do Ceará (IPECE) e do Instituto Brasileiro de Geografia e Estatística (IBGE). Na metodologia utilizou-se técnicas de análise multivariada: análise fatorial e análise de agrupamento ou cluster. Com base no cálculo do IVS, pôde-se concluir que a maioria dos municípios cearenses se enquadram na faixa de alta vulnerabilidade em saúde e uma minoria pertencem aos grupos de médio e baixo IVS. As cidades do interior e mais afastadas da capital são as que possuem maior índice, trazendo à tona uma realidade presente em muitas localidades no Brasil. Além de aprimorar e expandir o acesso à saúde, torna-se pertinente reduzir as desigualdades de renda, buscando também melhoria da infraestrutura nos municípios. Cabe ao poder público, por meio da elaboração de políticas públicas e deteç̧ão de falhas que travam o bom funcionamento do sistema, reduzir a vulnerabilidade em saúde existente no estado.

Palavras-chave: Ceará; Saúde; Vulnerabilidade.

\section{Vulnerability analysis of health services in the state of Ceará}

When the State promotes Health Services access, this parameter plays a vital role in promoting or revealing sustainable development and reducing socia vulnerability, primarily determined by environmental, economic, and social factors. This paper aims to analyze the health vulnerability in the State of Ceará, using the Health Vulnerability Index ( $\mathrm{HVI})$ as an indicator. The specific objectives are: to characterize the State of Ceará concerning health vulnerability indicators, formed from the variables selected for the study and calculate the HVI to enable the grouping and hierarchization of these cities about this index. The information was obtained through DATASUS, the Institute of Research and Economic Strategy of Ceará (IPECE), and the Brazilian Institute of Geography and Statistics (IBGE). Multivariate analysis techniques composed the methodology: factor analysis and cluster analysis. The calculation of the HVI concluded that the majority of Ceara cities get high ranges of health vulnerability, a minority belong to the groups of medium and low HVI. Towns in the rural area and furthest from the capital have the highest rates, rising a reality that is from Ceará and other locations in Brazil. In addition to improving and expanding access to health, it is pertinent to reduce income inequalities and improve services' infrastructure in municipalities. It is up to public authorities the responsibility and obligation to elaborate and implement public policies that will detect the failures that compromise the health system in to reduce the health high vulnerability ranges in the State of Ceará.

Keywords: Ceará; Health; Vulnerability.

Topic: Desenvolvimento, Sustentabilidade e Meio Ambiente

Reviewed anonymously in the process of blind peer.
Received: 01/04/2021

Approved: 28/04/2021

Gerlânia Maria Rocha Sousa

Universidade Federal do Ceará, Brasil http://lattes.cnpq.br/1718482251358328

http://orcid.org/0000-0001-6778-1946

gerlaniarocha@gmail.com

Maria Elisa Zanella (iD)

Universidade Federal do Ceará, Brasil

http://lattes.cnpq.br/4796364766536684

http://orcid.org/0000-0003-3475-2705

elisazv@gmail.com

\section{Referencing this:}

SOUSA, G. M. R.; ZANELLA, M. E.. Análise da vulnerabilidade em saúde no estado do Ceará. Revista Ibero Americana de Ciências Ambientais, v.12, n.4, p.472-488, 2021. DOI: http://doi.org/10.6008/CBPC21796858.2021 .004 .0037 


\section{INTRODUÇÃO}

A Constituição Federal de 1988, em seu artigo 196, garante o direito universal à saúde sob a responsabilidade do Estado, através do Sistema Único de Saúde (SUS). Ao dispor sobre organização e funcionamento dos serviços de saúde, na lógica de um sistema único, a Legislação Brasileira, em 1990, já concebia que as condições de promoção, proteção e recuperação da saúde envolvessem fatores como alimentação, trabalho, renda, saneamento básico, acesso a bens, serviços e informação (CARMO et al., 2018).

Ao longo do tempo vem ampliando-se às discussões acerca dos determinantes que interferem na saúde dos indivíduos. Buss et al. (2017) em um estudo mais recente afirmam que os Determinantes Sociais da Saúde são os fatores sociais, econômicos, culturais, étnicos/raciais, psicológicos e comportamentais que influenciam a ocorrência de problemas de saúde e seus fatores de risco na população.

É amplamente reconhecido que os resultados de saúde têm uma intensa associação com a situação socioeconômica dos indivíduos, tornando-se mais evidente em comunidades desiguais e áreas urbanas densas. A condição de saúde também é influenciada por fatores sociais, ambientais, políticos, econômicos e culturais. Esses fatores acrescentam qualidade de moradia, acesso a saúde e educação, trabalho, qualidade ambiental, disponibilidade de saneamento e segurança (OLIVEIRA et al., 2019).

Se a doença é manifesta num indivíduo, então ela é espacialmente localizada. Por sua vez, seja uma cidade ou uma região inteira, os lugares são o resultado de um acúmulo de eventos históricos, ambientais e sociais que promovem condições particulares à geração de doenças (BARCELLOS et al., 2002). Nesse sentido, um dos pontos importantes às análises ditas conjunturais acerca da saúde em ambientes de alta vulnerabilidade, diz respeito ao desenvolvimento de indicadores que possam demonstrar sob quais condições colocam a saúde dos indivíduos em risco.

Ademais, pode-se dizer que a saúde também é determinada por fatores ambientais, econômicos e sociais, sendo um importante indicador destas três dimensões que fazem parte do desenvolvimento sustentável. Nesse caso, cabe ao Estado, por meio de um conjunto de políticas públicas, a difícil tarefa de equilibrar desenvolvimento e equidade social, caso contrário, indivíduos e localidades estarão em um permanente e implacável regime de vulnerabilidade.

Esse entendimento é amplamente destacado por vários autores. Por exemplo, a seu modo, Dantas et al. (2016) entende que a vulnerabilidade é uma noção multidimensional, proveniente de fenômenos diversos, cujas causas e consequências são distintas, que afeta, de forma diferenciada, pessoas e grupos sociais. Uma vez estabelecidas, as condições sociais, culturais, étnicas, políticas, econômicas, educacionais e de saúde expõem às pessoas, em geral, a gozarem de um estado de bem-estar ou, em caso contrário, as submetem, não obstante a toda sorte de dificuldades e riscos.

No Brasil, a distribuição de recursos para o financiamento da saúde, e demais políticas sociais comprometidas com a justiça social e a melhoria da qualidade de vida, tem sido uma demanda da sociedade e um desafio constantemente lançado aos gestores públicos. Pobreza, definida por baixa renda, analfabetismo e baixa escolaridade, más condições de habitação e assistência inadequada à saúde expressam 
as condições de vida de várias famílias, mais frequentemente em zonas rurais e áreas urbanas irregulares (DRACHLER et al., 2014).

Por apresentar este caráter interdisciplinar de análise, essa temática não se limita apenas a uma determinada linha de pesquisa. Profissionais de diversas áreas, como por exemplo, geógrafos, economistas, sociólogos, gestores ambientais, dentre outros, compartilham desta discussão e tem desenvolvido trabalhos buscando identificar condições que comprovem estas relações, de forma que seja possível indicar ações para melhoria da qualidade de vida das populações.

A área de estudo deste trabalho compreende o estado do Ceará que é formado por 184 municípios e possui uma área total de $148.886,3 \mathrm{~km}^{2}$, sendo o 4 ㅇ maior estado da região Nordeste e o 170 entre os estados do Brasil em termos de extensão territorial. O mesmo é composto por 14 Regiões de Planejamento, sendo às mesmas: Cariri, Centro Sul, Grande Fortaleza, Litoral Leste, Litoral Norte, Litoral Oeste / Vale do Curu, Maciço de Baturité, Serra da Ibiapaba, Sertão Central, Sertão de Canindé, Sertão dos Crateús, Sertão dos Inhamuns, Sertão de Sobral e Vale do Jaguaribe (IPECE, 2019).

Nestes termos, o objetivo geral deste trabalho é analisar a vulnerabilidade em saúde dos municípios que compõem o Ceará. Ademais, os objetivos específicos são: caracterizar o estado com relação a indicadores de saúde selecionados e calcular o Índice de Vulnerabilidade em Saúde (IVS), de modo a possibilitar o agrupamento e a hierarquização dos municípios com relação a vulnerabilidade em saúde.

\section{MATERIAIS E MÉTODOS}

\section{Fonte de dados e dos indicadores}

Os dados deste trabalho foram obtidos através do banco de dados do Instituto de Pesquisa e Estratégia Econômica do Ceará (IPECE), do DATASUS e do Instituto Brasileiro de Geografia e Estatística (IBGE). Foram utilizados dados referentes ao ano de 2010 e para algumas variáveis utilizou-se informações de 2012, 2015, 2016, 2018 e 2019. Esses últimos seriam as informações mais atuais referentes a algumas variáveis.

As informações foram operacionalizadas por meio do software estatístico SPSS 25.0 e foram utilizadas 20 variáveis para a determinação do nível de vulnerabilidade em saúde dos municípios pertencentes ao Ceará (Quadro 1). As variáveis são referentes a indicadores socioeconômicos, de infraestrutura e situação de saúde no âmbito municipal.

Para a determinação das variáveis, utilizou-se como base o World Risk Report (JESCHONNEK, 2013) que formulou 28 indicadores para o cálculo do World Risk Index relacionando risco e vulnerabilidade em saúde. Também se tomou como base os 18 indicadores utilizados para a construção do índice de vulnerabilidade da saúde elaborado pela prefeitura de Belo Horizonte em 2012 (PITCHON et al., 2018). Outro trabalho que ajudou na seleção dos indicadores foi desenvolvido por Chan et al. (2019) que elaborou um índice de vulnerabilidade da saúde para redução de risco de desastres na região Belt and Road Initiative (BRI), também conhecida como a região "Silk Road Economic Belt". Inspirou-se também para as escolhas das variáveis a pesquisa desenvolvida por Oliveira et al. (2019) que formulou um índice de vulnerabilidade 
socioeconômica em saúde em Portugal.

Por fim, para a elaboração das variáveis também se utilizou indicadores de saúde elaborados pelo IPECE para o 'Perfil das Regiões de Planejamento' do estado, criado para possibilitar uma abordagem regional no planejamento das políticas públicas estaduais.

Tabela 1: Variáveis selecionados para a construção do Índice de Vulnerabilidade em Saúde (IVS).

\begin{tabular}{|c|c|c|}
\hline Variáveis & Ano & Fonte \\
\hline População residente por domicílio & 2012 & IPECE \\
\hline Percentual da população com rendimento per capita menor do que $1 / 4$ do salário mínimo & 2010 & IBGE \\
\hline Índice de gini & 2010 & IBGE \\
\hline Taxa de analfabetismo & 2010 & IBGE \\
\hline Destino do lixo inadequado ou ausente & 2010 & IPECE \\
\hline Domicílios particulares sem energia elétrica & 2012 & IPECE \\
\hline Abastecimento de água inadequado ou ausente & 2016 & IPECE \\
\hline Esgotamento sanitário inadequado ou ausente & 2016 & IPECE \\
\hline Recursos transferidos do SUS/habitante & 2019 & DATASUS \\
\hline Despesa total com saúde/habitante & 2019 & DATASUS \\
\hline Profissionais de saúde ligados ao SUS - agentes comunitários de saúde & 2015 & IPECE \\
\hline Profissionais de saúde ligados ao SUS - dentistas & 2015 & IPECE \\
\hline Profissionais de saúde ligados ao SUS - enfermeiros & 2015 & IPECE \\
\hline Profissionais de saúde ligados ao SUS - médicos & 2015 & IPECE \\
\hline Unidades de saúde ligadas ao SUS por municípios & 2015 & IPECE \\
\hline Leitos ligados ao SUS por municípios & 2015 & IPECE \\
\hline Internações por municípios & 2019 & DATASUS \\
\hline Taxa de cobertura de imunizações por município & 2019 & DATASUS \\
\hline Óbitos por município & 2018 & DATASUS \\
\hline Taxa de mortalidade infantil neonatal por município & 2016 & IPECE \\
\hline
\end{tabular}

Para a realização da análise da vulnerabilidade em saúde foram empregadas as técnicas de análise fatorial e a análise de agrupamento ou cluster, técnicas estatísticas de análise multivariada de dados. Trabalhos realizados com metodologias semelhantes foram desenvolvidos por Guimarães et al. (2014), Amendola et al. (2017), Damasceno (2018), IPECE (2018), Nayak et al. (2018), Azevedo et al. (2019), Chan et al. (2019), Hung et al. (2019) e Oliveira et al. (2019).

\section{Análise fatorial}

Existem diferentes propostas metodológicas para a construção de índices sintéticos, porém, nesta pesquisa adotou-se a análise fatorial pois a mesma é tida como um procedimento mais completo e sofisticado para esse tipo de cálculo. Conforme Fávero et al. (2009) o método de análise fatorial consiste na tentativa de se determinar as relações quantitativas entre as variáveis, aferindo seus padrões de movimento, de modo a associar àquelas um padrão semelhante, o efeito de um fator causal subjacente e específico a estas variáveis.

Essa análise baseia-se na suposta existência de um número de fatores causais gerais, cuja presença dá origem as relações entre as variáveis observadas, de forma que, no total, o número de fatores seja consideravelmente inferior ao número de variáveis. O modelo matemático (simplificado) da análise fatorial pode ser representado por:

$$
Z_{j=} \sum a_{j i} F_{i_{+}} d_{j} u_{j}(j=1,2, \ldots, n) ;(i=1,2, \ldots, m)
$$

Tal que:

$Z_{j=\text { j-ésima variável padronizada; }}$ 


$$
\begin{array}{r}
a_{j i} \text { = é o coeficiente de saturação referente ao i-ésimo fator comum da j-ésima variável; } \\
F_{i} \text { = é o i-ésimo fator comum; } \\
d_{j} \text { = é o coeficiente de saturação referente ao j-ésimo fator específico da j-ésima variável; } \\
u_{j}=\text { é o j-ésimo fator específico da j-ésima variável. }
\end{array}
$$

De acordo com a análise fatorial, cada fator é constituído por uma combinação linear das variáveis originais inseridas no estudo. A associação entre fatores e variáveis se dá por meio das cargas fatoriais, os quais podem ser positivos ou negativos, mas nunca superiores a um. Esses coeficientes de saturação têm função similar aos coeficientes de regressão na análise (HAIR et al., 2009).

O coeficiente de saturação entre uma variável e um fator elevado ao quadrado identifica a proporção da variância da variável explicada pelo fator. E o somatório do quadrado dos coeficientes de saturação, para cada variável, é chamado "comunalidade", a qual informa a proporção da variância total de cada variável, que é explicada pelo conjunto de fatores considerados na análise, ao passo que a soma do quadrado dos coeficientes de saturação para cada fator denomina-se eigenvalue. Ao dividir o eigenvalue pelo número de variáveis incluídas no estudo, obtém-se a proporção explicada pelo referido fator ao problema estudado (HAIR et al., 2009).

O primeiro procedimento necessário é a verificação dos pressupostos que consistirá em analisar a normalidade da distribuição dos dados de cada variável (utilizando o Teorema do Limite Central, caso haja um grande número de variáveis aleatórias independentes e identicamente distribuídas, então a distribuição tenderá para uma distribuição normal, à medida que o número dessas variáveis aumentar indefinidamente), além da estimação da matriz de correlação para checar a existência de relação entre as variáveis realizada por meio de testes de hipóteses específicos (FÁVERO et al., 2009).

A análise da matriz de correlação apresenta os coeficientes de correlação de Pearson para cada par de variáveis adotadas na pesquisa. A relação entre as variáveis é confirmada a partir do nível de significância dos coeficientes estimados (p-value $<0,05$ ). De acordo com Hair et al. (2009), a análise é iniciada com exame da matriz de correlações para verificação da existência de valores significativos que justifiquem a utilização da técnica. Ainda segundo os autores, se a visualização da matriz de correlações não mostrar um número substancial de valores maiores que 0,30, haverá fortes indícios que a análise fatorial não será adequada.

Conforme Fávero et al. (2009) para verificar a adequabilidade dos dados para a análise fatorial, são utilizados o índice Kaiser - Mayer - Olkin (KMO), o teste de esfericidade de Bartlett e a Matriz Anti -imagem. Em relação à estatística do $\mathrm{KMO}$, quanto menor o valor do respectivo teste, menor a relação entre as variáveis e os fatores, podendo o índice variar entre 0 e 1.0 teste Bartlett pode testar a hipótese nula de que a matriz de correlações é uma matriz identidade (o que inviabiliza a metodologia da análise fatorial proposta). Caso a matriz de correlações seja uma matriz identidade, significa que as inter-relações entre as variáveis são iguais a zero e, portanto, a análise fatorial não deverá ser utilizada, sendo $H_{0}$ (a matriz de correlações é uma matriz identidade) e $H_{a}$ (a matriz de correlações não é uma matriz identidade). Caso $H_{0}$ for aceito, a análise fatorial deve ser desconsiderada, caso $H_{0}$ seja rejeitado, haverá indícios de que existam correlações entre as 
variáveis explicativas utilizadas.

A matriz anti-imagem também mostra, a partir da matriz de correlações, a adequabilidade dos dados à análise fatorial e apresenta os valores negativos das correlações parciais. Na sua diagonal são apresentados os valores de MSA (Measure of Sampling Adequacy) ou a Medida de Adequação da Amostra, para cada variável, ou seja, quanto maiores esses valores, melhor será a utilização da análise fatorial e, caso contrário, talvez seja necessário excluí-la (HAIR et al., 2009).

A Análise dos Componentes Principais (ACP) leva em conta a variância total dos dados e, na análise fatorial comum os fatores são estimados levando-se em conta apenas a variância comum. O ACP se aplica quando o objetivo da análise for reduzir o número de variáveis para a obtenção de um número menor de fatores necessários a explicar o máximo possível a variância representada pelas variáveis originais.

O procedimento utilizado neste trabalho levou em consideração a extração dos fatores iniciais através da ACP que mostrou uma combinação linear das variáveis observadas, de maneira a maximizar a variância total explicada. A escolha do número de fatores se deu através do critério da raiz latente (critério de Kaiser) em que se escolheu o número de fatores a reter, em função dos valores próprios acima de 1 (eigenvalues) que mostram a variância explicada por cada, ou quanto cada fator conseguirá explicar da variância total (FÁVERO et al., 2009).

A grande dificuldade ao se optar pela análise fatorial é a interpretação dos fatores. Nem sempre se consegue identificar claramente quais variáveis estão sendo mais bem explicadas por um fator. Uma forma de minimizar essa dúvida é aplicar o método de rotação. Para a rotação dos fatores será utilizado o método varimax que é o mais utilizado e minimiza o número de variáveis com altas cargas em diferentes fatores permitindo a associação de uma variável a um único fator (FÁVERO et al., 2009).

Por fim, os fatores foram interpretados e nomeados através das cargas fatoriais. Segundo Fávero et al. (2005), considera-se que as cargas fatoriais maiores que 0,30 atingem o nível mínimo; cargas de 0,40 são consideradas mais importantes; cargas superiores a 0,50 são consideradas estatisticamente significativas. As variáveis com cargas mais elevadas influenciam mais a seleção de um nome ou rótulo para representar um fator.

\section{Cálculo do índice de vulnerabilidade em saúde (IVS)}

Através dos fatores extraídos e das suas respectivas cargas fatoriais, obtidos através da aplicação da análise fatorial, foi possível estudar o nível de vulnerabilidade em saúde nos municípios do estado do Ceará. Com base na metodologia proposta por Lemos (2000), foi calculado o IVS para os municípios. O índice foi calculado a partir dos escores estimados associados aos fatores obtidos na estrutura fatorial definida. Dessa forma, os fatores extraídos foram agregados a partir da seguinte equação:

$$
I V S_{i}=\sum_{j=1}^{n} \frac{\lambda_{j}}{\sum \lambda_{j}} F_{j i}^{*}
$$


$F_{j i}^{*}$ é o j-ésimo escore fatorial do i-ésimo município $\sum \lambda_{j}$ é o somatório das raízes características referentes aos $\mathrm{n}$ fatores extraídos. $\frac{\lambda_{j}}{\sum \lambda_{j}}$ se refere à participação relativa do fator $\mathrm{j}$ na explicação da variância total captada pelos $n$ fatores extraídos. A padronização dos escores fatoriais torna-se necessária de forma a enquadrá-los no intervalo de 0 a 1 , a partir da

$$
F_{j i}^{*}=\frac{F j i-F \min }{\text { Fmax }-F \min }
$$

Sendo que: $F_{j i}^{*}$ : escore fatorial do j-ésimo fator padronizado do i-ésimo município; $F_{j i}$ : escore fatorial do j-ésimo fator para o j-ésimo município; Fmin: menor escore fatorial do j-ésimo fator entre os municípios; Fmax: maior escore fatorial do j-ésimo fator entre os municípios. O IVS agrega seis importantes dimensões da vulnerabilidade em saúde no estado do Ceará (destacadas abaixo) e tem variação entre 0 (valor mínimo) e 1 (valor máximo).

\section{Agrupamento dos municípios com características semelhantes quanto à vulnerabilidade em saúde no Ceará: análise de agrupamento ou cluster}

Procedeu-se, ainda, à aplicação de outra técnica de estatística multivariada, a Análise de Agrupamento ou Cluster, a qual consiste na definição de grupos homogêneos e/ou heterogêneos, constituindo-se em um método orientador e norteador para identificação de diferenças de comportamento, tomada de decisões e definição de estratégias de atuação e planejamento.

O método adotado foi a Análise de Agrupamento Não Hierárquico (Técnica de Partição ou Agrupamento de k-médias), recurso comumente utilizado em estudos exploratórios descritivos, de modo a permitir uma classificação das comunidades rurais do município de acordo com a mensuração do capital social, tendo sido necessária a definição do número de agrupamentos. “O método de k-médias é responsável por alocar cada um dos elementos existentes em um dos k grupos pré-definidos, objetivando minimizar a soma dos quadrados residuais dentro de cada grupo com a finalidade de aumentar a homogeneidade do mesmo" (FÁVERO et al., 2009).

Consiste, portanto, em dividir um conjunto de elementos (municípios) em subconjuntos, os mais semelhantes possíveis, de modo que os elementos pertencentes a um mesmo grupo sejam similares com respeito às características que forem medidas em cada elemento. Ou seja, através de tal procedimento estatístico, os elementos são classificados em grupos restritos homogêneos internamente, com variabilidade intraclasse mínima e interclasse máxima (HAIR et al., 2009).

Os grupos foram divididos com base nos valores obtidos para o IVS a partir da análise fatorial, conforme definido anteriormente. Neste trabalho, os municípios do Ceará foram divididos (pelo pesquisador) em três clusters, para melhor compor a análise, sendo classificados da seguinte forma mostrado na tabela 2.

Tabela 2: Classificação dos municípios cearenses de acordo com o intervalo do ISVS definido pela análise de cluster.

\begin{tabular}{lllll}
\hline Clusters (Faixas do IVS) & $\begin{array}{l}\text { Intervalo } \\
\text { ISVS }\end{array}$ & $\begin{array}{l}\text { do } \\
\text { Municípios }\end{array}$ & $\begin{array}{l}\text { de } \\
\text { Estudados }\end{array}$ & $\begin{array}{l}\text { dos } \\
\text { Municípios }\end{array}$ \\
\hline $\begin{array}{l}\text { Municípios com menores níveis de vulnerabilidade em saúde } \\
\text { Municípios com níveis intermediários de vulnerabilidade em }\end{array}$ & $0,28 \mid-0,39$ & 10 & $6 \%$ \\
& $0,40 \mid-0,47$ & 40 & $22 \%$ \\
$\begin{array}{l}\text { saúde } \\
\text { Municípios com maiores níveis de vulnerabilidade em saúde }\end{array}$ & $0,48 \mid-0,62$ & 134 & $72 \%$ \\
\hline
\end{tabular}




\section{RESULTADOS E DISCUSSÃO}

\section{Análise da vulnerabilidade em saúde: análise fatorial}

Em relação aos pressupostos da análise fatorial, com o intuito de verificar se os dados suportam a análise foi feita a análise da matriz de correlação entre as variáveis, com o teste estatístico de esfericidade de Barlett. O valor do teste é 1365,129 com nível de significância ( $p$-value $=0,000$ ) (é recomendado que o valor da significância não ultrapasse 0,05$)$, nesse caso pode-se rejeitar a hipótese nula, ao nível de 1\%, de que a matriz de correlação é uma identidade, evidenciando, portanto, que há correlações entre as variáveis, tornando possível a aplicação do método. Para identificar a adequabilidade da análise fatorial, foi utilizado o índice de KMO. O resultado desse teste foi 0,772 (aproximadamente $77 \%$ ), indicando assim uma correlação boa entre as variáveis e evidenciando que a técnica é adequada.

Através do método dos componentes principais foram extraídos seis fatores com autovalor maiores do que 1 e eles em conjunto explicam aproximadamente $64,43 \%$ da variância dos dados originais, sendo este um percentual significativo (Tabela 3).

Tabela 3: Fatores extraídos pelo método dos componentes principais.

\begin{tabular}{llll}
\hline Fator & Autovalor & $\begin{array}{l}\text { Variância Explicada } \\
\text { Inicial }\end{array}$ & Rotação \\
\hline $\mathbf{1}$ & & 23,653 & 17,895 \\
$\mathbf{2}$ & 4,731 & 14,968 & 16,217 \\
$\mathbf{3}$ & 2,994 & 7,884 & 9,945 \\
$\mathbf{4}$ & 1,577 & 6,807 & 7,472 \\
$\mathbf{5}$ & 1,361 & 5,936 & 7,360 \\
$\mathbf{6}$ & 1,187 & 5,178 & 5,538 \\
Total & 1,036 & 64,427 & 64,427 \\
\hline
\end{tabular}

Para Silva et al. (2016), as comunalidades representam a proporção da variância para cada variável incluída na análise que é explicada pelos componentes extraídos. Geralmente o valor mínimo aceitável é de 0,50. A baixa comunalidade entre um grupo de variáveis indica que elas não estão linearmente correlacionadas e, por isso, não devem ser incluídas na análise em questão. No caso desta pesquisa (tabela 4) todas as variáveis apresentam comunalidades com valores acima de 0,5.

Também foi feita a rotação ortogonal dos fatores através do método Varimax para distinguir os valores das cargas fatoriais, de forma que cada variável fique conectada a um fator, facilitando a interpretação. Após a rotação, os fatores, em conjunto, continuam explicando $64,43 \%$ da variância total. 0 fator 1 é o mais importante do conjunto e explica 17,895\% da variância. Sendo assim, o modelo estimado mostrou que a vulnerabilidade em saúde no Ceará é explicada por seis fatores. As cargas fatoriais com valor superior a 0,50 evidenciam as variáveis mais fortemente associadas a determinado fator (Tabela 4).

Tabela 4: Matriz de cargas fatoriais do modelo fatorial estimado e comunalidades.

\begin{tabular}{|c|c|c|c|c|c|c|c|}
\hline Variáveis & Fator 1 & Fator 2 & Fator 3 & Fator 4 & Fator 5 & Fator 6 & Comunalidades \\
\hline População residente por domicílio & & & 0,580 & & & & 0,543 \\
\hline $\begin{array}{l}\text { Percentual da população com rendimento per } \\
\text { capita menor do que } 1 / 4 \text { do salário mínimo }\end{array}$ & & 0,876 & & & & & 0,850 \\
\hline Índice de gini & & 0,569 & & & & & 0,698 \\
\hline Taxa de analfabetismo & & 0,822 & & & & & 0,797 \\
\hline Destino do lixo inadequado ou ausente & & 0,719 & & & & & 0,595 \\
\hline
\end{tabular}


Domicílios particulares sem energia elétrica

Abastecimento de água inadequado ou ausente

Esgotamento sanitário inadequado ou ausente

Recursos transferidos do SUS/habitante

Despesa total com saúde/habitante

Profissionais de saúde ligados ao SUS - ACS

Profissionais de saúde ligados ao SUS - dentistas

Profissionais de saúde ligados ao SUS -

enfermeiros

Profissionais de saúde ligados ao SUS - médicos

Unidades de saúde ligadas ao SUS por municípios

Leitos ligados ao SUS por municípios

Internações por municípios

Taxa de cobertura de imunizações por município

Óbitos por município

Taxa de mortalidade infantil neonatal por município
0,549

0,579

$0,940 \quad 0,864$

$0,546 \quad 0,522$

0,717

0,795

0,596

0,580

0,555

0,800

0,693

0,753

0,618

0,588

0,670

0,531

0,590

0,524

0,707

0,533

0,696

0,807

0,686

Com base na tabela acima pode-se afirmar que o Fator 1 se relaciona mais fortemente com as variáveis recursos transferidos do SUS/habitante, despesa total com saúde/habitante e profissionais de saúde ligados ao SUS - médicos. De forma mais moderada, também mantém relacionamento com as variáveis leitos ligados ao SUS por município e profissionais de saúde ligados ao SUS - enfermeiros. Sendo assim, este fator pode ser denominado como indicador de recursos financeiros e acesso a atendimento de saúde.

Conforme discutido no World Risk Report (JESCHONNEK, 2013) um dos maiores problemas relacionados a saúde que alguns países enfrentam (principalmente os países do Sul global), estão relacionados a falta de financiamento de serviços de saúde. Para os indivíduos com baixa renda, o pagamento de determinados serviços de saúde pode se tornar um obstáculo para a melhoria de sua situação de saúde. A Organização Mundial da Saúde (OMS) estima que 100 milhões de pessoas no mundo são levadas a pobreza porque precisam arcar com gastos de saúde.

Para Damasceno (2018), as questões relacionadas a financiamento e gestão de recursos financeiros do SUS são problemáticas. O subfinanciamento é considerado o aspecto mais grave do sistema. Os recursos financeiros para a saúde devem ser disponibilizados de maneira adequada e eficiente de modo a garantir melhoria e acesso aos serviços prestados.

Uma das grandes problemáticas do Ceará e da saúde pública no Brasil é a carência de médicos e outros profissionais de saúde, além da má distribuição destes profissionais, acarretando desigualdade na oferta e no atendimento. A questão da disponibilidade de leitos pelo SUS também é um fator que contribui para a vulnerabilidade em saúde. Para Godoy et al. (2018) Fortaleza tem maior capacidade na prestação de atendimentos mais complexos e em relação ao Brasil, só fica atrás de São Paulo e Rio de Janeiro, porém, ainda há condições inadequadas de serviços de atendimentos e serviços públicos de saúde. Há uma sobrecarga de atendimentos e busca por leitos em unidades hospitalares da capital, que é intensificado pelo limitado número e pela alta demanda e precariedade dos serviços públicos de saúde no interior. A instalação dos hospitais regionais da região Cariri em Juazeiro do Norte, da região Sertão Central em Quixeramobim e da região Norte em Sobral, com o objetivo de absorver o fluxo de pacientes dessas regiões que seriam transferidos para Fortaleza, foi insuficiente, os mesmos possuem falhas administrativas, carência de 
profissionais especializados, somados a ampla demanda advinda de outras regiões.

O Fator 2 explica 16,21 \% da variação comum e apresenta uma forte relação com as variáveis percentual da população com rendimento per capita menor do que $1 / 4$ do salário mínimo, taxa de analfabetismo e destino do lixo inadequado ou ausente. De forma mediana esse fator também se relaciona com índice de gini e domicílios particulares sem acesso à energia elétrica. Sendo assim, pode-se denominar este fator de indicador de pobreza, escolaridade e situação de domicílio.

A distribuição de renda é considerada um determinante da desigualdade em saúde, ou seja, um fator que gera vulnerabilidade nesse âmbito. Sousa et al. (2016) apresentam resultados que demonstram que um aumento na renda per capita ocasiona um efeito positivo na Taxa de Mortalidade Infantil (TMI). Para os autores, a redução da pobreza traz contribuições para o declínio da TMI e melhoria na situação de saúde e qualidade de vida das famílias.

Em uma pesquisa elaborada por Houghton et al. (2020) em quatro países (Colômbia, El Salvador, Paraguai e Peru), concluiu-se que o percentual de indivíduos que enfrentam barreiras ao acesso a serviços de saúde foi elevado, especialmente entre pessoas de baixa renda, mesmo com reformas no setor de saúde. O progresso misto nas tendências de desigualdade reflete a complexidade e a multidimensionalidade do acesso aos cuidados de saúde.

A falta de escolaridade e a baixa renda dos indivíduos tendem a elevar os indivíduos a maiores níveis de vulnerabilidade em saúde. Conforme informações do Jornal Diário do Nordeste (2019) através de dados da plataforma integrasus, quanto menor o grau de escolaridade, maior é a TMI. Em 2019, dos 48.749 óbitos registrados, 4.749 foram de pessoas sem qualquer formação estudantil, o equivalente a $30,25 \%$ do total. As doenças do aparelho circulatório, como o infarto e o Acidente Vascular Cerebral (AVC), lideraram o ranking das cinco principais causas de mortalidade neste ano. Quanto menor o índice de escolaridade, menor o acesso à leitura e, por consequência, o acesso à informação e o entendimento do que é importante para a sustentação da saúde.

No que diz respeito ao destino do lixo inadequado ou ausente, Oliveira et al. (2019) debate sobre a contribuição negativa deste indicador para o meio ambiente, pois o lixo quando coletado de forma inadequada é colocado no mesmo recipiente, sem qualquer diferenciação de tipo de material, e depois descartado em aterros sanitários. Amaral et al. (2015) destacam que para garantir a sustentabilidade por meio da coleta de lixo, deve-se haver uma frequência na prestação deste serviço, e o material coletado deve ter um destino adequado. Não adianta coletar o lixo e despejá-lo em um local inadequado, como os lixões, que irão promover degradação ambiental, mau cheiro, além de vetores de doenças.

Para Oliveira (2018), quanto ao tipo de coleta de lixo empregado na zona urbana e rural das cidades cearenses, foi possível observar que na primeira ocorreu a prevalência da coleta de lixo por meio de serviço de limpeza e nas zonas rurais um maior número de queimadas dos resíduos na propriedade onde o material foi gerado. $\mathrm{O}$ autor explica que o crescimento populacional nos últimos anos provocou uma elevação na geração de lixo e o estado não conta com locais adequados para destinação final dos resíduos de várias localidades, sendo ainda predominante os chamados "lixões". 
Sobre a variável energia elétrica, Sena (2017) salienta que a ausência de energia elétrica prejudica o uso de equipamentos médicos, refrigeração de medicamentos e vacinas, suspensão de atendimentos e serviços hospitalares e aumento da demanda de atendimentos e suprimentos dos serviços de saúde em outras localidades, que são solicitadas para atender os indivíduos dessas áreas afetadas.

O Fator 3 está fortemente associado as variáveis unidades de saúde ligadas ao SUS por município, população residente, profissionais de saúde ligados ao SUS - agentes comunitários de saúde e dentistas. Assim, este fator pode ser denominado de indicador de população e acesso a serviços de saúde. O mesmo contribui para explicar 9,95\% da variação comum.

A atenção primária da saúde trabalha na promoção da saúde de forma ampla, através das unidades de saúde que contam com equipe multiprofissional que atuam em todos os atributos da atenção primária (coordenação do cuidado, primeiro contato com o sistema de saúde, integralidade, orientação familiar e comunitária, cuidado continuado) (SILVA et al., 2016). A atuação de profissionais como os agentes comunitários de saúde e dentistas, são de extrema importância, promovendo assim a prevenção da saúde dos indivíduos não doentes, porém expostos a fatores de risco para o desenvolvimento de doenças.

No estudo de Neves et al. (2019) constatou-se que existem importantes diferenças nas equipes de saúde bucal no Brasil, sendo que as regiões Sul e Sudeste apresentam as maiores prevalências de procedimentos odontológicos e as regiões Norte e Nordeste apresentaram as menores prevalências. A questão social tem sido evidenciada na questão da saúde bucal em várias populações de diferentes países. Estudos realizados tanto no nível individual quanto em grupos comprovam a relação entre desigualdades sociais, situação de vulnerabilidade e condições de saúde bucal.

O fator 4 apresenta uma forte relação com as variáveis taxa de cobertura de imunizações por município e taxa de mortalidade infantil neonatal por município e, por isso, foi denominado de indicador de imunizações e mortalidade infantil, explicando 7,47\% da variação comum. Para Chan et al. (2019) a variável imunizações é um bom indicador de desempenho do sistema de saúde. Em seu trabalho sobre vulnerabilidade em saúde e risco de desastres os autores concluíram que alguns países analisados, como por exemplo a Ucrânia, o país mais vulnerável da Europa, dentro da análise, possuía a taxa de vacinação mais baixa entre todos os que faziam parte do grupo estudado, devendo sua vulnerabilidade principalmente a esse fator. Em comunidades com baixas taxas de vacinação, surtos de doenças infecciosas podem acontecer, gerando uma sobrecarga no sistema de saúde.

No que diz respeito a TMI, conforme dados da SESA, a TMI no Ceará foi de 11 óbitos a cada 1.000 nascidos vivos em 2018 e nos últimos 5 anos esse índice permaneceu estável. Os relatos apontam para um cenário ainda preocupante e com falhas na assistência, desde o pré-natal até a hora do parto e os cuidados com o recém-nascido. $O$ estado possui assistência no pré-natal, porém, falta qualidade (ações que possam evitar complicações no parto e após o nascimento), há também falta de assistência adequada nas maternidades distribuídas pelas regiões e carência de especialistas no interior do estado.

Sousa et al. (2016) em seu estudo sobre a TMI nos estados do Brasil, analisam que mesmo com a atuação dos governos estaduais através de políticas públicas de saúde, como por exemplo, o Programa Saúde 
da Família (PSF) e o Programa de Agentes Comunitários de Saúde (PACS), voltados para a atenção primária da saúde, essa taxa ainda é considerada alta. Percebe-se assim, que o baixo número de profissionais de saúde no grupo com alto índice, a falta de especialistas e as deficiências estruturais, corroboram para a elevação dessa taxa.

Sobre o Fator 5, o mesmo tem forte relação com as variáveis internações e óbitos por município e pode ser denominado de indicador de internações e óbitos. Este fator explica 7,36\% da variação comum. A respeito das internações, Damasceno (2018) afirma que diversos trabalhos nacionais e internacionais mostram que elevadas taxas de internações por condições sensíveis à atenção básica estão associadas às dificuldades de acesso aos serviços básicos de saúde e à qualidade desses serviços. Conforme Elias et al. (2008) a amplitude das internações hospitalares por algumas causas selecionadas reflete a qualidade da atenção ou as linhas de cuidado oferecidas aos indivíduos, avaliando elementos de estrutura e processo das unidades de saúde e equipes de saúde associadas com essa atenção. Para os autores, nos últimos anos, temse observado o esforço político em adotar a avaliação como prática regular e sistemática de suas ações.

Sobre os óbitos, para Oliveira et al. (2019) as comunidades portuguesas que possuíam um maior número de mortes apresentaram maior vulnerabilidade em saúde, sendo que essas comunidades apresentaram um maior nível de desigualdade social. As comunidades com menores índices de mortalidade possuíam serviços especializados de saúde e recursos humanos, obtendo assim, melhores resultados de saúde. A OMS analisou os determinantes da saúde e uma de suas constatações foi que os grupos desfavorecidos são mais vulneráveis a doenças, morrem mais jovens e experimentam piores condições de saúde.

Por fim, o Fator 6 relaciona-se com as variáveis abastecimento de água inadequado ou ausente e esgotamento sanitário inadequado ou ausente, explicando 5,54\% da variação comum. Este fator pode ser descrito como indicador de acesso a água e esgotamento sanitário. Conforme exposto pela Fiocruz (MAIA, 2017), em 2007 a ONU declarou o acesso à água e ao esgotamento sanitário como um direito humano fundamental e uma pesquisa realizada na Fiocruz Minas mostra que a violação dessa prerrogativa afeta uma série de outros direitos sociais, como educação e saúde. Populações com precariedade no acesso à água e ao esgotamento acabam sendo excluídas de outras esferas da vida social e econômica.

O acesso à água potável e segura, mesmo sendo um direito humano básico, não tem sido estendido a todos os indivíduos, especialmente àqueles encontrados em áreas periurbanas esquecidas pelas políticas públicas de saúde e saneamento. Nas áreas urbanas brasileiras havia, em 2000, aproximadamente 18 milhões de pessoas sem acesso ao abastecimento público de água, 93 milhões sem coleta adequada de esgotos sanitários e 14 milhões sem serviços de coleta de resíduos sólidos. O abastecimento de água, cada vez mais, tem preocupado e sido palco de debate dos gestores públicos, pois a falta de acesso a água tem sido considerada fator de risco à saúde, além de limitante ao desenvolvimento sustentável (RAZZOLINI et al., 2008).

O saneamento ambiental, que envolve as atividades básicas de esgotamento sanitário, limpeza pública, drenagem urbana e controle de vetores de importância sanitária, tem o abastecimento de água 
como atividade primordial. No Brasil, as doenças relacionadas a ausências no saneamento ambiental resultaram em 3,4 milhões de internações no país, no período de 1995 a 1999 . O não acesso ou acesso inadequado à água potável compromete os usos menos imediatos e as condições de higiene. Essas situações induzem à busca de água em fontes alternativas, de qualidade sanitária duvidosa e a condições inadequadas de transporte e armazenamento da água (RAZZOLINI et al., 2008).

\section{Índice de Vulnerabilidade em Saúde (IVS)}

Em razão da dificuldade para analisar, agrupar e hierarquizar os municípios do estado do Ceará com relação a vulnerabilidade em saúde empregando apenas os valores dos escores fatoriais foi utilizado o IVS, calculado a partir da agregação dos seis fatores extraídos. $O$ índice possibilitou classificar com maior precisão os 184 municípios, facilitando a identificação daqueles com maior e menor vulnerabilidade em saúde. As informações relacionadas à distribuição absoluta e relativa dos municípios segundo as faixas do IVS foram apresentadas na tabela 1. A figura 2 permite a visualização dos municípios cearenses segunda a vulnerabilidade em saúde e pode-se perceber a predominância dos municípios com alto IVS.

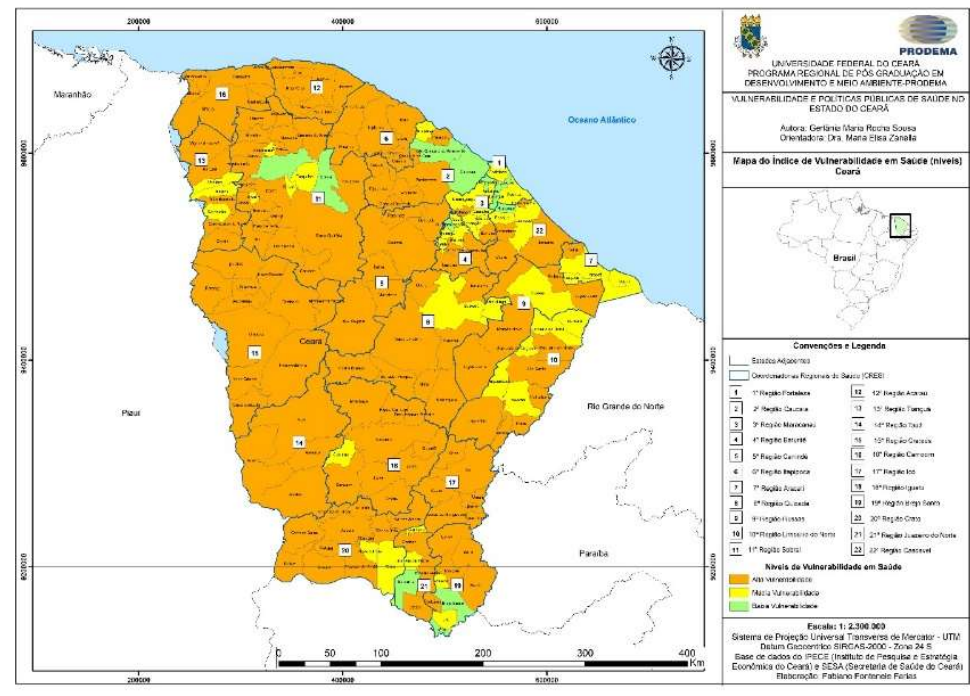

Figura 1: Classificação do IVS nos municípios do Ceará.

O IVS foi agrupado em três clusters e o cluster com menor nível de vulnerabilidade em saúde é o cluster 1 , destacando-se o município de Barbalha com IVS de 0,28. O cluster 2 com nível médio de vulnerabilidade em saúde foi representado por $22 \%$ dos municípios e o cluster 3 é composto pelos municípios com alto nível de vulnerabilidade em saúde, agrupando assim $72 \%$ das cidades cearenses, tendo destaque o município de Boa Viagem com IVS de 0,63, sendo o município com maior nível de vulnerabilidade em saúde do estado. É importante citar que o IVS do Ceará como um todo foi 0,51 enquadrando o estado na faixa de alto nível de vulnerabilidade em saúde. A tabela 5 apresenta um ranking dos municípios com os 10 maiores e os 10 menores IVS.

Tabela 5: Ranking dos municípios pelos 10 maiores e 10 menores ISVS.

\begin{tabular}{llllll}
\hline MUNICÍPIO & ISVS & RANKING 10 MAIORES & MUNICÍPIO & ISVS & RANKING 10 MENORES \\
\hline Boa Viagem & 0,63 & 1 & Barbalha & 0,28 & 1 \\
Ipueiras & 0,62 & 2 & Eusébio & 0,32 & 2
\end{tabular}




\begin{tabular}{llllrr} 
Ipaporanga & 0,61 & 3 & Guaramiranga & 0,34 & 3 \\
Mucambo & 0,60 & 4 & São Gonçalo do Amarante & 0,35 & 4 \\
Umari & 0,60 & 5 & Maracanaú & 0,35 & 5 \\
Viçosa do Ceará & 0,60 & 6 & Sobral & 0,35 \\
Granja & 0,60 & 7 & Penaforte & 0,36 \\
Parambu & 0,59 & 8 & Horizonte & 0,37 \\
Mombaça & 0,59 & 9 & Brejo Santo & 0,39 \\
Pereiro & 0,59 & 10 & Itaitinga & 0,40 & 10 \\
\hline
\end{tabular}

O município de Barbalha possui o menor IVS do estado e localiza-se na Região Metropolitana do Cariri. Conforme IPECE (2012) o município possui uma população de 56.576 habitantes. Para Damasceno (2018), a cidade tem destaque pelo elevado nível de investimento em saúde, transformando-a em um importante polo regional de saúde do estado, respondendo pela cobertura dos municípios da região Cariri do Ceará e de estados vizinhos. A autora cita que os hospitais públicos de Barbalha fazem parte da rede de assistência médica de alta complexidade do SUS e do governo do estado, fornecendo suporte ao Hospital Regional do Cariri em Juazeiro do Norte.

É válido salientar que na pesquisa sobre desenvolvimento da saúde no semiárido nordestino, Damasceno (2018) calculo o Índice de Desenvolvimento da Saúde (IDS) e através dos resultados percebeuse que Barbalha atingiu o segundo maior IDS, sendo considerado um município com elevado desenvolvimento em saúde. De acordo com dados da FIRJAN (2018), através do cálculo do Índice FIRJAN de Desenvolvimento Municipal (IFDM) a cidade obteve alto índice. O município também obteve alto desenvolvimento nas áreas de saúde e educação. Através do cálculo do Índice de Desenvolvimento Municipal (IDM), o IPECE (2018) destacou Barbalha como o único município da região Cariri que obteve elevado IDM.

No trabalho elaborado por Damasceno (2018) também se encontra com bons resultados dentro do semiárido nordestino o município de Sobral que se localiza na região noroeste do estado e Guaramiranga que está localizada na região nordeste. Os dados da FIRJAN (2018) revelam que Guaramiranga apresenta IFDM moderado, porém, se destaca com alto desenvolvimento nas áreas de educação e saúde.

No cluster 1 também estão inseridos cinco dos quinze municípios que compõem a Região Metropolitana de Fortaleza (RMF), sendo estes Eusébio, São Gonçalo do Amarante, Maracanaú, Horizonte e Itaitinga. Segundo números do IPECE (2019) Maracanaú, Sobral, São Gonçalo do Amarante, Eusébio e Horizonte fazem parte das dez maiores economias do estado em termos de Produto Interno Bruto (PIB).

Conforme dados da FIRJAN (2018), Eusébio foi a cidade melhor avaliada no Ceará em termos de desenvolvimento em saúde e educação. O IFDM também destacou Sobral, São Gonçalo do Amarante, Maracanaú, Horizonte e Itaitinga como tendo apresentado alto índices de desenvolvimento nas áreas de saúde e educação. O IPECE (2018) mencionou as cidades de São Gonçalo do Amarante, Eusébio, Maracanaú, Horizonte e Sobral como cinco dos dez melhores ranqueados no IDM do estado.

A cidade de Brejo Santo localizada na região Cariri foi destaque da área de educação, apresentando alto índice de desenvolvimento, de acordo com dados da FIRJAN (2018). No IDM calculado pelo IPECE (2018), o município obteve moderado desenvolvimento, porém, o mesmo destacou-se com bons resultados nas variáveis de saúde.

Em relação ao cluster 3, o mesmo possui como seu pior representante a cidade de Boa Viagem, 
localizada na microrregião dos Sertões de Canindé. Conforme informações do IPECE (2012), Boa Viagem possui uma população de 52.828 habitantes. Através da figura 1 nota-se que os municípios com baixa e média vulnerabilidade em saúde pertencem as regiões de saúde que compõem as seguintes regiões de planejamento: Grande Fortaleza, Litoral Leste, Maciço do Baturité, Vale do Jaguaribe, Cariri, Serra da Ibiapaba e Sertão de Sobral. As demais regiões (Centro Sul, Litoral Norte, Litoral Oeste/Vale do Curu, Sertão Central, Sertão de Canindé, Sertão dos Crateús, Sertão dos Inhamuns) comportam em sua maioria, os municípios com alto IVS.

É notório que os municípios com maiores índices fazem parte da região semiárida do Ceará. Para Damasceno (2018) o baixo desenvolvimento da saúde no semiárido brasileiro, ligado às condições sociais e econômicas adversas da região, implica que a realização de investimentos e o uso de estratégias para elevar a eficiência dos gastos são de suma importância, mas não são suficientes para garantir o desenvolvimento sustentável e uma maior qualidade de vida e bem-estar para os indivíduos.

A vulnerabilidade em saúde no estado do Ceará, constatada pelo alto IVS da maioria dos municípios, traz à tona uma realidade que se repete em muitas localidades no Brasil, principalmente em comunidades mais desfavorecidas. Nota-se que além de expandir o acesso à saúde, torna-se pertinente reduzir as desigualdades de renda entre os municípios, além da melhoria da infraestrutura. Dessa forma, acredita-se que uma possível forma para se minimizar no curto, médio e longo prazo é uma ação mais eficaz e conjunta de estados e municípios por meio dos mecanismos de gestão de saúde: secretarias, conselhos, fundos de saúde. Todavia, é preciso que o governo, como gestor público, promova melhorias nas políticas públicas de saúde, buscando detectar as falhas que travam o bom funcionamento desses projetos, principalmente no interior do estado.

A realidade demonstrada nesse trabalho contribui para os debates atuais e futuros sobre a questão da vulnerabilidade em saúde, podendo ser utilizado para o planejamento e/ou formulação de políticas públicas voltadas para o desenvolvimento dos municípios e melhoria das condições de vida dos indivíduos. Esta pesquisa ainda pode ser mais explorada, trazendo mais resultados para a análise da situação de vulnerabilidade em saúde das cidades cearenses. A metodologia ainda pode ser aplicada a realidade de outros estados, regiões e/ou país.

\section{CONCLUSÕES}

Existem diversos estudos sobre vulnerabilidade, no entanto ainda se nota uma escassez quando se trata de vulnerabilidade em saúde, principalmente envolvendo variáveis de saúde, socioeconômicas e de infraestrutura. Este artigo apresenta um índice de vulnerabilidade em saúde visando melhorar a análise da situação de saúde no Ceará. O IVS é composto por vinte variáveis e cobre seis indicadores. O trabalho ainda possui algumas limitações, discussões mais aprofundas a respeito dos indicadores e de políticas públicas de saúde são importantes para melhor compor os resultados.

Todavia, os resultados dessa pesquisa mostraram que no Ceará ainda há vários municípios com deficiências em relação a saúde e no que diz respeito a essa temática, pôde-se perceber que indicadores 
socioeconômicos e de infraestrutura corroboram para o avanço da vulnerabilidade na área da saúde. Dessa forma, tais dados e discussões servem para auxiliar o processo de elaboração de políticas públicas de saúde, focando nos indicadores que promovem vulnerabilidade e nos municípios com menores IVS.

\section{REFERÊNCIAS}

AMARAL, R. F.; CAMPOS, K. C.; LIMA, P. V. P. S.. Distribuição da pobreza no estado do Ceará: uma abordagem multidimensional. Interações, Campo Grande, v.16, p.327337, 2015.

DOI: https://doi.org/10.20435/interações.v16i2.70

AMENDOLA, F.; ALVARENGA, M. R. M. A.; LATORRE, M. R. D. O.; OLIVEIRA, M. A. C.. Family vulnerability index to disability and dependence (FVI-DD), by social and health conditions. Revista Ciência e Saúde Coletiva, Rio de Janeiro, v.22, p.112, 2017. DOI: http://dx.doi.org/10.1590/1413$\underline{81232017226.03432016}$

AZEVEDO, J. V. V.; SOUZA, P. M.; SANTOS, C. A. C.; SILVA, M. T.; SANTOS, D. A. S.. Aplicação da análise fatorial para determinação da vulnerabilidade socioeconômica e ambiental nos municípios do estado da Paraíba. RICA, v.10, p.97-111, 2019. DOI: https://doi.org/10.6008/CBPC2179$\underline{6858.2019 .004 .0008}$

BARCELLOS, C. C.; SABROZA, P. C.; PEITER, P.; ROJAS, L. I. Organização espacial, saúde e qualidade de vida: a análise espacial e o uso de indicadores na avaliação de situações de saúde. Informe Epidemiológico do SUS, Brasília, v.11, n.3, p.129-138, 2002

BEZERRA, R.. Maioria das mortes no Ceará é de pessoas sem escolaridade. Fortaleza: Jornal diário do Nordeste, 2019.

BUSS, P. M.; PELLEGRINI FILHO, A.. A saúde e seus determinantes sociais. PHYSIS: Revista de Saúde Coletiva, Rio de Janeiro, v.17, p.77-93, 2007. DOI: https://doi.org/10.1590/S0103-73312007000100006

CARMO, M. E.; GUIZARDI, F. L.. O conceito de vulnerabilidade e seus sentidos para as políticas públicas de saúde e assistência social. Cadernos de saúde pública, Brasília, v.34, p.1-14, 2018. DOI: https://doi.org/10.1590/0102-311x00101417

CHAN, E. Y. Y.; HUANG, Z.; LAM, H. C. Y; WONG, C. K. P.; ZOU, Q.. Health vulnerability index for disaster risk reduction: application in Belt and Road Initiative (BRI) region. International Journal of Environmental Research and Public Health, v.16, p.1-16, 2019. DOI: https://doi.org/10.3390/ijerph16030380

DAMASCENO, N. P.. Panorama da saúde pública no semiárido brasileiro: eficiência e desempenho. Tese (Doutorado) - Universidade Federal do Ceará, Fortaleza, 2018.

DANTAS, E. W. C.; COSTA, M. C. L.; ZANELLA, M. E.. Vulnerabilidade socioambiental e qualidade de vida em Fortaleza. Fortaleza: Imprensa Universitária, 2016.

DRACHLER, M. L.; DRACHLER, C. W.; LEITE, J. C. D. C.;
TEIXEIRA, L. B.; LOBATO, M. A. O.; LERMEN, J. I.; FAGUNDES S.; FERLA, A. A.. Desenvolvimento e validação de um índice de vulnerabilidade social aplicado a políticas públicas do SUS. Ciência e Saúde Coletiva, Rio de Janeiro, v.16, p.69-87, 2014. DOI: https://doi.org/10.1590/141381232014199.12012013

ELIAS, E.; MAGAJEWSKI, F.. A atenção primária à saúde no sul de Santa Catarina: uma análise das internações por condições sensíveis à atenção ambulatorial, no período de 1999 a 2004. Revista Brasileira de Epidemiologia, Florianópolis, v.11, p.633-647, 2008. DOI: https://doi.org/10.1590/S1415-790X2008000400011

FÁVERO, L. P.; BELFIORI, P.; SILVA, F. L.; CHAN, B. L.. Análise de dados: modelagem multivariada para tomada de decisões. Rio de Janeiro: Elsevier, 2009.

FIRJAN. Índice Firjan de desenvolvimento municipal (IFDM). Rio de Janeiro: FIRJAN, 2020

GUIMARÃES, R. M.; MAZOTO, M. L.; MARTINS, R. N.; CARMO, C. N.; ASMUS, C. I. F.. Construção e validação de um índice de vulnerabilidade socioambiental para a vigilância e gestão de desastres naturais no estado do Rio de Janeiro, Brasil. Revista Ciência e Saúde Coletiva, v.19, p.4157-4165, 2014. DOI: https://doi.org/10.1590/1413812320141910.08282014

GODOY, C. V.; SILVA, J. B.. A geografia urbana da saúde: influência regional dos serviços de saúde em Fortaleza CE/Brasil. Revista Caribeña de Ciências Sociales, v.10, p.117, 2018.

HAIR, F. J.; BLACK, W. C.; BABIN, B. J.; ANDERSON, R. E.; TATHAM, R. L.. Análise multivariada de dados. Porto Alegre: Bookman, 2009.

HOUGHTON, N.; BASCOLO, E.; RIEGO, A.. Las desigualdades economicas que representan obstaculos para buscar servicios de salud en cuatro paises de America Latina. Revista Panamericana de Salud Pública, v.44, p.1-16, 2020. DOI: https://doi.org/10.26633/RPSP.2020.11

IPECE. Instituto de Pesquisa e Estratégia Econômica do Ceará. As regiões de planejamento do estado do Ceará. Fortaleza: SEPLAG, 2012.

IPECE. Instituto de Pesquisa e Estratégia Econômica do Ceará. Índice de desenvolvimento municipal (IDM). Fortaleza: SEPLAG, 2018.

IPECE. Instituto de Pesquisa e Estratégia Econômica do Ceará. Painel de indicadores sociais e econômicos: os 10 maiores e os 10 menores municípios cearenses. Fortaleza: SEPLAG, 2018

IPECE. Instituto de Pesquisa e Estratégia Econômica do 
Ceará. Painel de indicadores sociais e econômicos: os 10 maiores e os 10 menores municípios cearenses. Fortaleza: SEPLAG, 2019.

JESCHONNEK, L.. World risk report 2013. Berlin: Alliance Development Works, 2013.

LEMOS, J. J. S.. Indicadores de Degradação no Nordeste Subúmido e Semiárido. RESR, Piracicaba, v.10, p.1-10, 2000.

OLIVEIRA, C. T. A.. Diagnóstico do gerenciamento de resíduos sólidos no estado do Ceará. Dissertação (Mestrado) - Universidade da Integração Internacional da Lusofonia Afro-Brasileira, Limoeiro do Norte, 2018.

OLIVEIRA, G. M.; VIDAL, D. G.; FERRAZ, M. P.; CABEDA, J. M.; PONTES, M.; MAIA, R. L.; CALHEIROS, J. M.; BARREIRA, E.. Measuring health vulnerability: an interdisciplinar indicator applied to mainland Portugal. International Journal of Environmental Research and Public Health, v.16, p.21-41, 2019. DOI: https://doi.org/10.3390/ijerph16214121

PITCHON, A.; GIRODO, A. M.; GOMES, C. G.; GOMES, D. H. P. G.. Índice de Vulnerabilidade da Saúde 2012. Belo Horizonte: Prefeitura municipal de Belo Horizonte, 2018.

MAIA, K.. Pesquisas mostram como o não acesso à água e ao esgoto afeta outros direitos sociais. Minas Gerais: Fiocruz Minas, 2017.
NEVES, M.; GIORDANI, J. M. A.; HUGO, F. N.. Atenção primária à saúde bucal no Brasil: processo de trabalho das equipes de saúde bucal. Revista Ciência e Saúde Coletiva, Rio de Janeiro, v.24, p.1-18, 2019. DOI:

https://doi.org/10.1590/1413-81232018245.08892017

RAZZOLINI, M. T. P.; GUNTHER, W. M. R.. Impacto na saúde das deficiências de acesso a água. Revista Saúde e Sociedade, v.17, p.1-10, 2008.

SENA, A. R. M.. Seca, vulnerabilidade, vulnerabilidade socioambiental e saúde: impactos no semiárido brasileiro. Tese (Doutorado) - Instituto de Comunicação e Informação Científica e Tecnológica em Saúde, Rio de Janeiro, 2017.

SILVA, B. P.; STOCKMANN, D.; LÚCIO, D. S.; HENNA, E.; ROCHA, M. C. P.; JUNQUEIRA, F. M.. Ampliação do acesso à saúde na região mais vulnerável do estado de São Paulo, Brasil: reflexo do Programa Mais Médicos? Revista Ciência e Saúde Coletiva, Rio de Janeiro, v.21, p.2899-2906, 2016. DOI: http://dx.doi.org/10.1590/141381232015219.15552016

SOUSA, J. S.; CAMPOS, R. T.; SILVA, A. F.. Estimação e análise dos fatores determinantes da redução da taxa de mortalidade infantil no Brasil. Revista Brasileira de Estudos Regionais e Urbanos (RBERU), Belo Horizonte, v.10, n.2, p.140-155, 2016.

A CBPC - Companhia Brasileira de Produção Científica (CNPJ: 11.221.422/0001-03) detém os direitos materiais desta publicação. Os direitos referem-se à publicação do trabalho em qualquer parte do mundo, incluindo os direitos às renovações, expansões e disseminações da contribuição, bem como outros direitos subsidiários. Todos os trabalhos publicados eletronicamente poderão posteriormente ser publicados em coletâneas impressas sob coordenação da Sustenere Publishing, da Companhia Brasileira de Produção Científica e seus parceiros autorizados. Os (as) autores (as) preservam os direitos autorais, mas não têm permissão para a publicação da contribuição em outro meio, impresso ou digital, em português ou em tradução. 